\title{
Percepção de faces em crianças e adolescentes com Transtorno Invasivo do Desenvolvimento ${ }^{1}$
}

\author{
Fernanda Tebexreni Orsati \\ Syracuse University, Syracuse-NY, Estados Unidos da América \\ Tatiana Mecca \\ José Salomão Schwartzman \\ Elizeu Coutinho de Macedo ${ }^{2}$ \\ Universidade Presbiteriana Mackenzie, São Paulo-SP, Brasil
}

\begin{abstract}
Resumo: Novas possibilidades promissoras de avaliação vêm sendo estudadas nos Transtornos Invasivos do Desenvolvimento (TID) como, por exemplo, análise dos movimentos oculares. O objetivo deste trabalho foi comparar crianças com TID e crianças com desenvolvimento normal na percepção de faces humanas, por meio da análise do rastreamento visual. Foram avaliados 10 participantes com TID, idade média de 11,9 anos $(D P=3,22)$, pareados por idade e sexo, com 10 crianças com desenvolvimento normal. Dez faces neutras em branco e preto foram observadas por 10 segundos, controlando-se nos estímulos: gênero (masculino ou feminino); posição (normal ou invertida) e presença dos olhos (presente ou ausente). Observaram-se diferenças significativas: o grupo TID olhou menos tempo para a região dos olhos nas figuras masculinas; gastou menos tempo na face e nos olhos em figuras invertidas; além de utilizar menos tempo na fixação da face com olhos presentes e ocultados; já o grupo controle olhou mais na região dos olhos, quando os estes estavam ocultados.
\end{abstract}

Palavras-chave: autismo, percepção da face, comportamento social.

\section{Face perception in children and adolescents with Pervasive Developmental Disorders}

\begin{abstract}
New promising assessment possibilities have been studied in Pervasive Developmental Disorders (PDD) such as eye tracking analysis. This study compares the perception of human faces in children with PDD and children with typical development through the analysis of eye-tracking patterns. Ten participants with PDD, mean age of 11.9 years $(S D=3.22)$, were matched by age and gender with 10 participants with typical development. Ten neutral faces, in black-and-white, were observed per 10 seconds each while some stimuli were controlled: gender (male or female); position (normal or upsidedown); presence of eyes (present or absent). Significant differences were found: PDD participants spent less time looking at eyes of male faces; spent less time with faces and eyes in upside-down figures and spent less time looking at faces with eyes and faces with occulted eyes; and control group spent more time looking at the eyes region when these were occulted.
\end{abstract}

Keywords: autism, face perception, social behavior.

\section{Percepción de rostros en niños y adolescents con Trastorno Invasivo del Desarrollo}

Resumen: Nuevas posibilidades de evaluación, como análisis de los movimientos oculares, estan siendo estudiadas en el Trastorno Invasivo del Desarrollo (TID). El objetivo de este trabajo fue comparar niños con TID y niños con desarrollo normal en la percepción de rostros humanos por medio de un análisis de rastreamiento visual. Fueron evaluados 10 participantes con TID, edad media de 11,9 años $(D P=3,22)$, pareados por edad y sexo con 10 niños controles. Fueron observados 10 rostros en blanco y negro por 10 segundos, controlandose: género (masculino o femenino); posición (normal o invertida); presencia de los ojos (presente o ausente). Se observaron diferencias significativas: el grupo TID miro menos para la región de los ojos en las figuras masculinas; gastaron menos tiempo en el rostro y en los ojos en figuras invertidas y gastaron menos tiempo en la fijación del rostro con ojos presentes y ocultos; y controles miraron más la región de los ojos en rostros con ojos ocultos.

Palabras clave: autismo, percepción facial, conducta social.

$\mathrm{O}$ autismo foi primeiramente descrito por Kanner (1943). Em seu trabalho, ele identificou 11 crianças, de 5 a 11 anos, possuindo o que denominou de Distúrbio Autístico

1 Este trabalho é parte da dissertação de mestrado da primeira autora orientada pelo último autor e contou com apoio dos órgãos de fomento à pesquisa CAPES, CNPq e Mackpesquisa. Este texto foi revisado seguindo Acordo Ortográfico da Língua Portuguesa (1990), em vigor a partir de $1^{\circ}$ de janeiro de 2009.

2 Endereço para correspondência:

Prof. Dr. Elizeu Coutinho de Macedo. Universidade Presbiteriana Mackenzie. Programa de Pós-graduação em Distúrbios do Desenvolvimento. Rua da Consolação, 896, $6^{\circ}$ andar, sala 62. CEP 01.302-907. São Paulo-SP, Brasil.E-mail: elizeumacedo@uol.com.br
Inato do Contato Afetivo. As crianças que Kanner descreveu apresentavam uma gama variada de sintomas com diferentes graus de comprometimento, tais como: falha em assumir postura para ser pego, dificuldade no ajustamento corporal no colo, atividades governadas por um desejo de isolamento e preservação da mesmice, preferência por relacionamento com objetos que não interferem neste isolamento e, quando inevitável, o estabelecimento de um relacionamento que tende a ser temporário.

Na Classificação Internacional de Doenças (CID-10) (Organização Mundial da Saúde [OMS], 1993), o autismo é enquadrado nos Transtornos Invasivos do Desenvolvimento (TID). Define-se TID como um grupo de transtornos 
caracterizados por alterações qualitativas das interações sociais recíprocas e modalidades de comunicação e por um repertório de interesses e atividades restrito, estereotipado e repetitivo. Estas anomalias qualitativas constituem uma característica global do funcionamento do sujeito, em todas as ocasiões, com aparecimento dos sintomas antes dos 36 meses (OMS, 1993). A estimativa de prevalência atual está entre 30 e 60 casos por 10.000 para o TID, sendo a proporção entre meninos e meninas de 4:1 (Fombonne, 2003; Yeargin-Allsopp e cols., 2003; Rutter, 2005).

Uma compreensão completa do quadro autístico envolve quatro níveis do conhecimento: etiologia, estruturas e processos cerebrais, neuropsicologia e sintomas e comportamento (Gadia, Tuchuman, \& Rotta, 2004). A análise dos movimentos oculares em estudos neuropsicológicos possibilita a compreensão dos mecanismos de controle de respostas motoras, bem como seu efeito diferencial nos distúrbios do desenvolvimento (Goldberg e cols., 2002). Estudos de anormalidades do movimento ocular expandem o conhecimento sobre processos complexos de maturação cerebral, de regulação genética e do desenvolvimento dos sistemas cerebrais complexos (Sweeney, Takarae, MacMillian, Luna, \& Minshew, 2004). Pesquisas vêm sendo realizadas no Brasil, demonstrando a importância de serem estudados os movimentos oculares em diferentes processos cognitivos, como em habilidades atencionais (Covre, Macedo, Capovilla, \& Schwartzman, 2005), habilidades de leitura e diversos distúrbios do neurodesenvolvimento como distúrbios psiquiátricos (Macedo e cols., 2005) e distúrbios invasivos (Mercadante, Macedo, Baptista, Paula, \& Schwartzman, 2006; Orsati, Schwartzman, Brunoni, Mecca, \& Macedo, 2008).

Dentre as pesquisas de anormalidades no movimento ocular no TID, um trabalho de revisão (Sweeney e cols., 2004) defende a importância do estudo de movimentos oculares na determinação de endofenótipos, definidos como fenótipos internos intermediários entre o gene e a condição em si (Bearden \& Freimer, 2006), e na investigação de aspectos cognitivos e neurofisiológicos. Tal trabalho relata ainda a importância de se estudar o processo de engajamento atencional, assim como os processos executivos de pessoas com autismo.

O estudo dos processos cerebrais complexos, assim como das funções cerebrais e suas características comportamentais relacionadas, é meio importante de se descobrir mais sobre os vários distúrbios neurológicos (Stahl, 2004). O estudo de anormalidades no movimento ocular é um método neurocientífico não invasivo e que dá pistas sobre o funcionamento cerebral. Portanto, os movimentos oculares são importantes em uma avaliação neurológica, pois seu exame avalia a distribuição dos circuitos neuronais e aponta para o comprometimento envolvido no processo (Stahl, 2004).

Duas vertentes na pesquisa de movimento ocular com população autista vêm sendo exploradas. A primeira verifica o padrão de fixações em relação a figuras sociais, para elucidação de como o autista explora seu ambiente. A segunda vertente analisa propriedades dinâmicas dos movimentos sacádicos, em diferentes tipos de tarefas (Sweeney e cols., 2004). Estudos que analisam os movimentos sacádicos descrevem dificuldades executivas na população com TID, caracterizadas principalmente por: falta de regulação da atenção voluntária; dificuldade em inibir um comportamento e direcioná-lo ao objetivo proposto na tarefa; e alteração no planejamento de ação e dificuldade para iniciação de resposta (Minshew, Luna, \& Sweeney, 1999; Luna, Doll, Minshew, \& Sweeney, 2007; Orsati e cols., 2008).

Nesse mesmo sentido, Klin, Jones, Schultz, Volkmar e Cohen (2002b) defendem que a análise do movimento ocular, no processamento de estímulos visuais sociais, representa uma promissora linha de pesquisa. Nesse estudo, os autores expuseram adultos normais e adultos autistas a cenas de filmes. Foram observados padrões diferentes de fixação entre os dois grupos, sendo que os indivíduos normais em uma cena de terror olharam mais para os olhos, já os autistas, para a boca. Autistas também demonstraram menor número de fixações nos rostos e menos sacadas entre os mesmos. Quando requeridos a julgar algumas pistas sociais sobre as personagens, eles apresentaram dificuldade de explicitar tal julgamento.

A percepção de faces promove informações ao indivíduo que facilitam sua interação social (Haxby, Hoffman, \& Gobbini, 2002). Partindo do pressuposto de que o reconhecimento de faces possa estar associado ao processo de sociabilidade, várias pesquisas têm sido conduzidas com a finalidade de comparar os padrões dos movimentos oculares na percepção de faces humanas, feitos por pessoas com autismo e controles. Tais pesquisas investigam, mas não são ainda conclusivas em afirmar que o fato de autistas olharem menos para a região dos olhos os tornem menos competentes socialmente (Klin, 2000; Klin, Jones, Schultz, Volkmar, \& Cohen, 2002a, 2002b; Pelphrey e cols., 2002; Mercadante e cols., 2006).

A vertente que estuda a percepção de faces parte da possibilidade de que o olhar direcionado à região dos olhos seja uma característica inicial da sociabilidade. Farroni, Csibra, Simion e Johnson (2002) observaram que bebês de cinco semanas preferem figuras de rostos humanos que olhem direto para elas comparados a figuras que desviam o olhar, e autistas com a mesma idade evitam o olhar direto da região dos olhos.

Em um estudo de análise do efeito da posição da face, Van der Geest, Kemner, Verbaten e Van Engeland (2002) observaram que crianças normais despendiam menos tempo em faces viradas de cabeça para baixo, mas que os autistas não apresentaram essa diferença. Tal disparidade foi correlacionada com o número e a duração das fixações oculares, demonstrando que crianças com desenvolvimento normal mantêm maior atenção em figuras sociais (faces na posição normal) e crianças com autismo mantêm maior atenção em estímulos não sociais (faces em posição invertida). Esses resultados parecem indicar que a falta de discriminação entre as orientações de faces esteja ligada à falta de processamento global de faces no autismo. 
Os estudos dos movimentos oculares em faces corroboram e complementam um estudo de Ressonância Magnética Funcional (Hadjikhani e cols., 2004). Esse estudo identifica algumas diferenças na ativação do giro fusiforme, entre outras áreas cerebrais, ligadas ao processamento de faces. Esses componentes do sistema de processamento de faces envolvem principalmente interpretação da informação, comunicada por meio de movimentos faciais como alternância do olhar e expressões emocionais, assim como o acesso ao significado afetivo e os sinais sociais que eles convergem.

O objetivo do presente trabalho é comparar o desempenho entre as crianças com TID e com desenvolvimento normal, no rastreio ocular de faces humanas.

\section{Método}

\section{Participantes}

Participaram do estudo 10 crianças e jovens do sexo masculino com TID, pareados por idade e sexo, com 10 crianças e jovens com desenvolvimento típico. A idade dos participantes variou entre 8 e 19 anos $(M=11,9 ; D P=3,22)$. O diagnóstico clínico de TID foi dado por um neurologista experiente, baseando-se nos critérios para Transtorno Invasivo do Desenvolvimento do DSM IV (American Psychiatric Association, 1994). Não foram incluídos participantes com comorbidades psiquiátricas ou neurológicas, assim como participantes em que não foi possível a calibração do equipamento de registro dos movimentos oculares pelo fato de estarem agitados.

Os critérios de padronização do diagnóstico de TID foram feitos a partir das pontuações mínimas para TID na Kiddie-Sads (K-SADS) adaptado para o Brasil (Mercadante e cols., 1995). Nesse instrumento, quanto maior a pontuação maior o número de características autísticas e, portanto, maior comprometimento de habilidades como interação social e comunicação. Todos os participantes obtiveram, no mínimo, quatro pontos que é a nota de corte para Transtorno Invasivo do Desenvolvimento. Todos os participantes avaliados foram capazes de compreender e seguir instruções verbais.

\section{Instrumentos}

A inteligência foi avaliada com a Escala de Inteligência Wechsler Infantil $3^{\mathrm{a}}$ edição (Figueiredo, 2001) composta por 14 testes, subdivididos em Escala Verbal e de Execução, e tem como objetivo avaliar o Quociente Geral de Inteligência (QI), medindo o potencial intelectual da criança.

Para a avaliação do desempenho do reconhecimento de emoções em expressões faciais, foi utilizada a versão adaptada do teste computadorizado de identificação de emoções faciais, Penn Emotion Acuity Test (PEAT), desenvolvido por Erwin e cols. (1992). PEAT é composto por 40 fotos em preto e branco de faces humanas de ambos os sexos, retratando expressão com conteúdo emocional feliz, triste e neutra. Foram selecionadas 24 fotos de faces humanas, com expressão neutra, sendo metade de cada sexo. Além disso, as fotos foram manipuladas em programa de editoração de imagem, sendo que metade delas foi rotacionada em $180^{\circ}$, para serem apresentadas como estímulo na posição invertida.

Do total de 24 fotos, 12 tiveram os olhos cobertos em cor cinza, tirando a impressão de olhar das faces. Assim, os 24 estímulos foram agrupados da seguinte forma: Gênero (Masculino ou Feminino); Posição (Normal ou Invertida); Presença dos Olhos (Presente ou Ausente). As dimensões dos estímulos variaram de 20 a 23 centímetros de comprimento e de 16 a 18 centímetros de largura.

Foi utilizado o equipamento computadorizado Tobii ${ }^{\circledR}$ 1750 (Tobii Technology) para o registro do padrão de movimentos oculares. $\mathrm{O}$ equipamento consiste em um monitor de vídeo, 17" TFT 1280x1024 pixels, que tem embutido na sua parte inferior microlâmpadas de diodos que emitem luz infravermelha, e duas câmeras de alta resolução que filmam ambos os olhos simultaneamente. Os raios infravermelhos incidem sobre os olhos do participante e refletem diretamente sobre as lentes das câmeras, possibilitando a identificação da direção do olhar e de sua localização em relação à tela do computador. $\mathrm{O}$ equipamento é calibrado de acordo com o tamanho da pupila e da curvatura da córnea de cada participante, a fim de garantir maior precisão na identificação dos pontos de fixação. Os participantes ficavam sentados, com os olhos a uma distância aproximada de 50 centímetros da tela do computador. Neste estudo não foi utilizado nenhum apoio para a cabeça, pois o equipamento é capaz de registrar os movimentos oculares, mesmo que haja movimento da cabeça, em qualquer direção, de até 5 centímetros.

\section{Procedimento}

Primeiramente era realizada uma entrevista inicial com os pais ou responsáveis pelas crianças, para levantamento de dados de identificação, histórico pessoal e familiar. Nesta mesma sessão, foi aplicado o instrumento diagnóstico K-SADS. Os responsáveis pelos participantes autorizaram a participação na pesquisa e assinaram o Termo de Informação e Consentimento Livre e Esclarecido, aprovado pelo Comitê de Ética em Pesquisa da Universidade Presbiteriana Mackenzie. Os participantes foram avaliados em duas sessões de aproximadamente 2 horas, sendo que a primeira consistiu na entrevista com os pais e na aplicação do WISC. Na segunda sessão, os participantes observavam as fotos do PEAT que eram apresentadas no equipamento de rastreio dos movimentos oculares. Com esse fim, sentavam-se à frente da tela do computador onde os estímulos eram apresentados e eram orientados a olhar livremente para os rostos. Cada estímulo com as faces era apresentado por 10 segundos na tela do computador, e o participante deveria simplesmente observá-la. Os participantes do grupo controle passaram pelas mesmas atividades e testes das crianças do grupo TID.

\section{Análise de dados}

Todas as medidas avaliadas foram tabuladas, descritas e posteriormente analisadas por meio do programa estatístico 
SPSS versão 13.0. A fim de comparar os desempenhos entre os grupos, foram conduzidas estatísticas não paramétricas pelo Teste Mann-Whitney para duas amostras independentes. A utilização de estatísticas não paramétricas deve-se ao fato de as medidas de inteligência e dos parâmetros dos movimentos oculares não apresentarem distribuição normal. Para comparação entre as variáveis intragrupo (estímulo Feminino versus Masculino; Invertido versus Normal e Olhos Presentes versus Olhos Ocultados), foi utilizada estatística não paramétrica de comparação entre duas amostras relacionadas, o Teste Wilcoxon. Foram considerados valores significativos para todas as variáveis, quando o índice $p$ indicou valor igual ou menor que 0,05 .

\section{Resultados}

A avaliação de inteligência revelou que o Quociente de Inteligência (QI) do grupo TID $(M=77,33 ; D P=22,14)$ foi significativamente inferior $(U=9,0 ; p=0,00)$ ao do grupo controle $(M=115,40 ; D P=11,77)$. Análises estatísticas de comparação entre os grupos pelo Teste Mann-Whitney indicaram diferenças significativas para o QI Verbal $(U=5,5 ; p=0,00)$ e QI de Execução $(U=19 ; p=0,02)$. Apesar da diferença entre os grupos, o QI de Execução do grupo TID ( $M=90,44$; $D P=23,21)$ encontra-se dentro da faixa do que é esperado para a população geral que varia entre 85 e 115 pontos.

$\mathrm{Na}$ Tarefa de Rastreamento de Faces, foram avaliadas as seguintes variáveis em todos os estímulos: número de fixações na região dos olhos, número de fixações na face, tempo de fixação na região dos olhos e tempo de fixação na face (em segundos). A Tabela 1 apresenta os resultados gerais da Tarefa de Rastreamento de Faces.

Tabela 1

Médias e desvio-padrão do número e tempo de fixação, na região dos olhos e face, por grupo

\begin{tabular}{lccccc}
\hline & & TID & Controle & $\boldsymbol{U}$ & $\boldsymbol{p}$ \\
\hline N Fixações & $\mathrm{M}$ & 7,56 & 8,96 & \multirow{2}{*}{30,50} & 0,14 \\
Face (F) & $\mathrm{DP}$ & 1,68 & 1,87 & & \\
N Fixações & $\mathrm{M}$ & 3,99 & 4,76 & \multirow{2}{*}{31,00} & 0,17 \\
Olhos (O) & $\mathrm{DP}$ & 1,22 & 1,08 & & \\
Tempo Fixação & $\mathrm{M}$ & 2,83 & 3,33 & \multirow{2}{*}{17,00} & $0,01^{*}$ \\
Face & $\mathrm{DP}$ & 0,56 & 0,19 & & \\
Tempo & $\mathrm{M}$ & 1,56 & 1,95 & & \\
Fixação Olhos & $\mathrm{DP}$ & 0,57 & 0,56 & 37,00 & 0,35 \\
\hline
\end{tabular}

* valor de $p \leq 0,05$

Observa-se diferença entre os grupos no tempo de fixação na face $(p=0,01)$, sendo que os participantes com TID fixaram aproximadamente 0,5 segundo a menos do que os controles nesta região. Não foram encontradas diferenças significativas para os números de fixações em nenhuma das comparações realizadas. Desta forma, a seguir são analisados apenas os dados de tempo de fixação.
A fim de verificar o efeito do tipo de estímulo, foram conduzidas análises estatísticas entre os grupos para cada um dos tipos de estímulos usados. Foi encontrada diferença no tempo de fixação nas faces para as seguintes variáveis: Masculino $(p=0,04)$, Invertido $(p=0,02)$ e Olhos Presentes $(p=0,05)$ e Ocultados $(p=0,01)$, sendo que o grupo controle despendeu mais tempo do que o grupo TID em todos os estímulos. Foi também encontrada diferença na região dos olhos, em relação à face, para a variável Invertido $(p=0,04)$, sendo que o grupo controle despendeu mais tempo na região dos olhos, quando as faces estavam invertidas. Tais resultados estão sumariados na Tabela 2, bem como as comparações entre os dois grupos para as variáveis: Gênero (Masculino e Feminino), Posição (Normal e Invertido) e Presença dos Olhos (Presente e Ausente).

Tabela 2

Médias e desvio-padrão do tempo de fixação, na região dos olhos e face, para cada uma das variáveis: Gênero, Posição e Presença dos Olhos

\begin{tabular}{lcccccccc}
\hline & \multicolumn{4}{c}{ Face } & \multicolumn{5}{c}{ Face } \\
\cline { 2 - 9 } & TID & Controle & $\boldsymbol{U}$ & $\boldsymbol{p}$ & TID & Controle & $\boldsymbol{U}$ & $\boldsymbol{P}$ \\
\hline Masculino & 2,85 & 3,35 & 22,50 & $0,04^{*}$ & 1,59 & 1,97 & 33,50 & 0,22 \\
Feminino & 2,82 & 3,25 & 28,00 & 0,11 & 1,55 & 2,00 & 36,00 & 0,31 \\
Normal & 2,88 & 3,30 & 32,00 & 0,19 & 1,73 & 1,94 & 34,50 & 0,25 \\
Invertido & 2,76 & 3,32 & 19,00 & $0,02^{*}$ & 1,23 & 1,93 & 22,00 & $0,04^{*}$ \\
Olhos & 2,84 & 3,25 & 24,50 & $0,05^{*}$ & 1,47 & 1,84 & 36,50 & 0,31 \\
Presentes & & & & & & & & \\
Olhos & 2,83 & 3,43 & 17,00 & $0,01^{*}$ & 1,76 & 2,15 & 33,00 & 0,22 \\
Ocultados & & & & & & & &
\end{tabular}

* valor de $p \leq 0,05$

A Figura 1, Figura 2 e Figura 3 exemplificam as interações entre grupos, encontradas na tarefa de Rastreamento de Faces. A figura é gerada pela compilação das médias de todas as fixações realizadas na figura apresentada, para cada grupo. A face é o estímulo, as áreas contornadas pelas cores claras, bem como as áreas claras, demonstram locais em que houve pontos de fixação, sendo que as áreas contornadas por linhas claras ilustram porções da figura com maior concentração de pontos de fixação e as áreas claras demonstram menor concentração de pontos de fixação no local. Portanto, as áreas com contornos claros indicam as regiões que os sujeitos mais olharam.

Na Figura 1, comparando-se os estímulos com os registros dos movimentos oculares dos dois grupos, observa-se uma área bem definida na região dos olhos correspondente às fixações desta região, bem como alguns pontos que representam fixações isoladas. Assim, o padrão de fixação, na região dos olhos, é muito semelhante nos dois grupos. No entanto, no grupo TID, observam-se menos fixações na região do nariz e boca em comparação com o grupo controle, indicando que os participantes com TID olharam menos para estas regiões. Além disso, observa-se que os participantes com TID apresentaram número maior de pontos de fixações isolados e até mesmo fora da região das faces. 


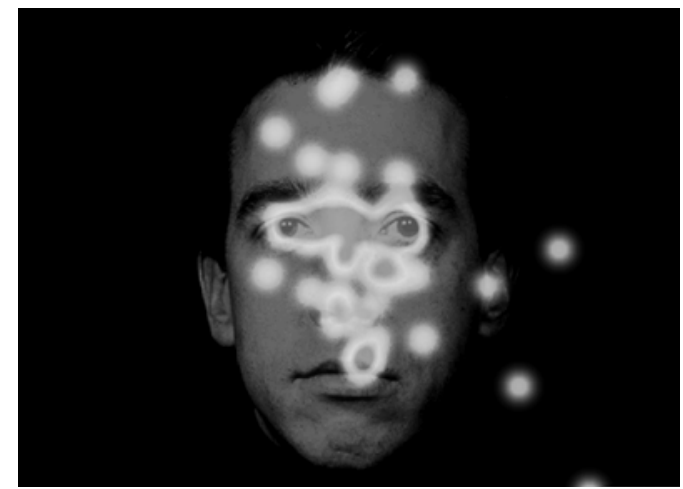

Grupo TID

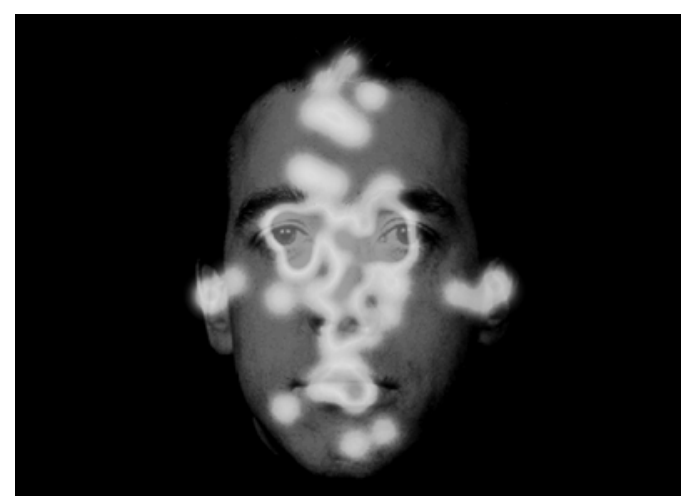

Grupo Controle

Figura 1. Exemplo da compilação de pontos de fixação por grupo para a variável Masculino (maior tempo de fixação na face masculina para grupo controle).

A Figura 2 ilustra as fixações dos participantes do grupo controle e TID, durante a inspeção visual de faces invertidas. A mancha maior na região dos olhos do grupo controle indica que eles olharam significativamente mais

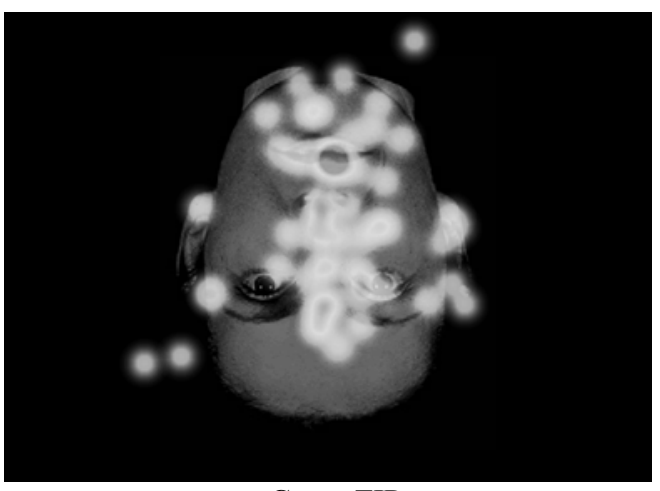

Grupo TID para essa região do que os participantes com TID, tal como descrito na Tabela 2. Além disso, participantes com TID apresentaram muitos pontos de fixação dispersos, alguns até fora da face.

Figura 2. Exemplo da compilação de pontos de fixação por grupo para a variável Invertido (maior tempo de fixação na face e na região dos olhos para grupo controle).

A Figura 3 ilustra o padrão de fixações dos dois grupos de participantes, durante a observação de faces com os olhos removidos. Com relação à localização das fixações na face, observa-se um padrão semelhante nos dois

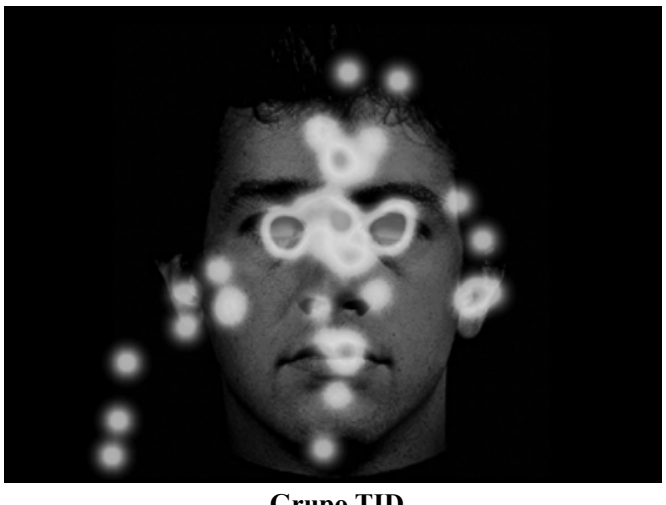

Grupo TID grupos, concentradas principalmente na região dos olhos e boca. Além disso, observa-se que os participantes do grupo TID realizaram fixações mais dispersas e fora da face.

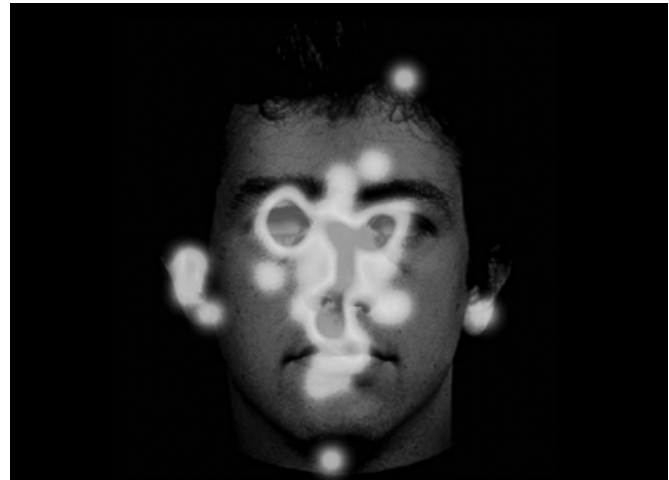

Grupo Controle

Figura 3. Exemplo da compilação de pontos de fixação por grupo para a variável Olhos Ocultados (maior tempo de fixação na face para grupo controle). 
Após a análise entre os grupos, teste Wilcoxon para duas amostras relacionadas foi conduzido. Tal análise procurou verificar o efeito dos pares de variáveis: Gênero (Feminino versus Masculino), Posição (Normal versus Invertida) e Presença de Olhos (Olhos Presentes versus Ocultados) de cada grupo separadamente. Para o grupo TID, foi encontrada diferença no tempo de fixação na região dos olhos das faces Normais e Invertidas $(Z=-2,59 ; p=0,00)$, sendo que o grupo TID apresenta um tempo de fixação maior para faces Normais. Para os participantes do grupo controle, foi encontrada diferença significativa para o tempo de fixação na região dos olhos para as faces com Olhos Presentes e Ocultados $(Z=-2,29 ; p=0,02)$, sendo o tempo de fixação maior nas figuras com Olhos Ocultados.

\section{Discussão}

Não foram encontradas diferenças significativas entre o número de fixações na região dos olhos, como descrito na literatura para faces na posição normal entre participantes com TID e participantes com desenvolvimento normal (Klin, 2000; Klin e cols., 2002a; Klin e cols., 2002b; Pelphrey e cols., 2002; Mercadante e cols., 2006). A ausência de diferença no número de fixações, na região dos olhos, pode ser em função de os estímulos apresentados neste estudo terem sido de faces sem cor e movimento, diminuindo os efeitos de um estímulo social natural, assim como o menor tempo de apresentação das figuras.

Encontrou-se diferença para o tempo de fixações em toda a face entre os grupos, sendo que o grupo TID apresentou menor tempo de fixação. Esse dado demonstra que participantes com TID apresentam dificuldade em manter a atenção e olham menos para a figura como um todo. Se a observação de faces possibilita obter informações que facilitam a interação social (Haxby, Hoffman, \& Gobbini, 2002), essas crianças e jovens que estão despendendo menos tempo do que seus pares na exploração de rostos humanos não obtêm informações necessárias para boa compreensão do estímulo e, consequentemente, apresentam mais dificuldades na interação social.

A manipulação de outras variáveis, como presença de olhos e posição do rosto, também pode contribuir para entendimento desses mecanismos perceptuais e atencionais nas pessoas com TID. Van der Geest e cols. (2002) encontraram que pessoas com TID apresentam mais fixações em faces invertidas em relação a faces normais. Tal diferença não foi encontrada no presente trabalho. Os participantes com TID do presente estudo apresentaram menor tempo de fixação nas faces e na região dos olhos para as faces invertidas, quando comparados aos participantes do grupo controle. Ou seja, os participantes com TID da presente amostra não demonstraram ter preferência pelas faces invertidas, como apresentado previamente na literatura.

No entanto, os participantes do grupo controle apresentaram maior número de fixações na região dos olhos, quando os mesmos estão ocultados, diferença não encontrada no grupo TID. A ausência do efeito da remoção dos olhos nos participantes com TID pode ser devido ao fato de que, no presente estudo, os olhos foram removidos dos estímulos, mas as regiões dos mesmos permaneceram e podiam dar a impressão de que as pessoas estivessem com os olhos fechados. Desta forma, as diferenças entre as figuras com e sem olhos podem não ter sido tão evidentes e improváveis, como seria esperado.

Outra hipótese que pode ser levantada é que, de acordo com Van der Geest e cols. (2002), sujeitos com desenvolvimento típico percebem a figura de rosto como um todo e dirigem sua atenção para regiões específicas de figuras que parecem incomuns. No caso das faces apresentadas, o estímulo incomum seria uma face humana sem os olhos. Possivelmente, os participantes com TID, por não perceberem a face como um todo, não identificam as partes ausentes e não focam sua atenção na mesma. Esse dado pode ser correlacionado com o de Van der Geest e cols. (2002) que analisaram o processamento de faces invertidas. Naquele trabalho, a ausência de identificação de figuras invertidas por pessoas com TID também é explicada em função da ausência de processamento global de faces no autismo. Em suma, o fato de os participantes não fixarem o olhar com mais intensidade na região incomum da figura, falta de olhos, quer dizer que eles não estão processando a mesma de uma maneira global, assim como os participantes do grupo controle, e percebendo o estímulo incomum da mesma.

\section{Considerações finais}

O presente trabalho não corrobora dados da literatura que descrevem que participantes com TID evitam a região do olhar, ou preferem faces invertidas. Os presentes achados indicam que participantes com TID apresentam padrão de exploração de faces diferente dos participantes com desenvolvimento normal. Esta diferença se caracteriza por apresentarem menos tempo de fixação nas faces, acarretando a obtenção de menos informações necessárias para interações sociais. Outro resultado encontrado é a diferença na percepção da região dos olhos, quando estes estão ausentes, indicando dificuldade na percepção global de faces por pessoas com TID.

Enfim, hipotetiza-se que uma percepção de estímulos sociais que não seja eficaz pode gerar dificuldade na compreensão de situações sociais o que, por consequência, pode gerar comportamentos socialmente inadequados.

\section{Referências}

American Psychiatric Association. (1994). Diagnostic and statistical manual of mental disorders: DSM-IV. Washington: Author.

Bearden, C. E., \& Freimer, N. B. (2006). Endophenotypes for psychiatric disorders: Ready for primetime? Trends Genet, 22, 306-13. 
Covre, P., Macedo, E. C., Capovilla, F. C., \& Schwartzman, J. S. (2005). Movimentos oculares e padrões de busca visual em tarefas de rotação mental. Psico-USF, 10, 41-49.

Erwin, R. J., Gur, R. C., Gur, R. E., Skolnick, B., MawhinneyHee, M., \& Smailis, J. (1992). Facial emotion discrimination: I. Task construction and behavioral findings in normals. Psychiatry Research, 42, 231-240.

Farroni, T., Csibra, G., Simion, F., \& Johnson, M. H. (2002). Eye contact detection in human from birth. Proceedings of the National Academy of Sciences, 99, 9602-9605.

Figueiredo, V. L. M. (2001). WISC III: Escala de inteligência Wechsler para crianças (3a ed.). São Paulo: Casa do Psicólogo.

Fombonne, E. (2003). Epidemiological surveys of autism and other pervasive developmental disorders: An update. Journal of Autism and Developmental Disorders, 33, 365-382.

Gadia, C. A., Tuchman, R., \& Rotta, N. T. (2004). Autismo e doenças invasivas de desenvolvimento. Jornal de Pediatria, 80, 83-94.

Goldberg, M. C., Lasker, A. G., Zee, D. S., Garth, E., Tien, A., \& Landa, R. J. (2002). Deficits in the initiation of eye movements in the absence of a visual target in adolescents with high functioning autism. Neuropsychologia, 40, 2039-2049.

Hadjikhani, N., Joseph, R. M., Snyder, J., Chabris, C. F., Clark, J., Steele, S., McGrath, L., Vangel, M., Aharon, I., Feczko, E., Harris, G. J., \& Tager-Flusberg, H. (2004). Activation of the fusiform gyrus when individuals with autism spetrum disorder view faces. Neuroimage, 22, 1141-1150.

Haxby, J. V., Hoffman, E. A., \& Gobbini, M. I. (2002). Human neural system for faces recognition and social communication. Biological Psychiatry, 51, 59-67.

Kanner, L. (1943). Autistic disturbances of affective contact. Nervous Child, 2, 217-250.

Klin, A. (2000). Attributing social meaning to ambiguous visual stimuli in higher-functioning autism and Asperger syndrome: The social attribution task. Journal Child Psychology and Psychiatry, 41, 831-46.

Klin, A., Jones, W., Schultz, R., Volkmar, F., \& Cohen, D. (2002a). Defining and quantifying the social phenotype in autism. American Journal of Psychiatry, 159, 895-908.

Klin, A., Jones, W., Schultz, R., Volkmar, F., \& Cohen, D. (2002b). Visual fixation patterns during viewing of naturalistic social situations as predictors of social competence in individuals with autism. Archives of General Psychiatry, 59, 809-816.

Luna, B., Doll, S. K., Minshew, N. J., \& Sweeney J. A. (2007). Maturation of executive function in autism. Biological Psychiatry, 61, 474-481.
Macedo, E. C., Lukasova, K., Macedo, G. C., Duduchi, M., Capovilla, F. C., \& Schwartzman J. S. (2005). Avaliação neuropsicológica e análise dos movimentos oculares na esquizofrenia. In E. C. Macedo \& F. C. Capovilla (Orgs.), Temas em neuropsicolinguística (pp. 21-38). Ribeirão Preto, SP: Tecmedd.

Mercadante, M. T., Asbahr, F., Rosário, M. C., Ayres, A. M., Ferrari, M. C., Assumpção, F. B., \& Miguel, E. C. (1995). Versão brasileira: Eugenia Deheinzelin KSADS. Entrevista semi-estruturada para diagnóstico em psiquiatria da infância: Versão epidemiológica. Manuscrito não-publicado, São Paulo.

Mercadante, M. T., Macedo, E. C., Baptista, P. M., Paula, C. S., \& Schwartzman, J. S. (2006). Saccadic movements using eye-tracking technology in individuals with autism spectrum disorders: Pilot study. Arquivos de NeuroPsiquiatria, 64, 559-562.

Minshew, N. J., Luna, M. D., \& Sweeney, J. A. (1999). Oculomotor evidence for neocortical systems but not cerebellar dysfunction in autism. Neurology, 52, 917922.

Organização Mundial da Saúde. (1993). CID-10: Classificação Estatística Internacional de Doenças $e$ Problemas Relacionados à Saúde (10a ed.). São Paulo: EDUSP.

Orsati, F., Schwartzman, J. S., Brunoni, D., Mecca, T., \& Macedo, E. C. (2008). Novas possibilidades na avaliação neuropsicológica dos transtornos invasivos do desenvolvimento: Análise dos movimentos oculares. Revista Avaliação Psicológica, 7, 281-290.

Pelphrey, K. A., Sasson, N. J., Reznick, J. S., Paul, G., Goldman, B. D., \& Piven, J. (2002). Visual scanning of faces in autism. Journal of Autism and Developmental Disorders, 32, 249-61.

Rutter, M. (2005). Autism research: Lessons from the past and prospects for the future. Journal of Autism and Developmental Disorders, 35, 241-57.

Stahl, J. S. (2004). Using eye movements to assess brain function in mice. Vision Research, 44, 3401-10.

Sweeney, J. A., Takarae, Y., MacMillian C., Luna, B., \& Minshew, N. J. (2004). Eye movements in neurodevelopmental disorders. Current Opinion in Neurology, 17, 37-42.

Van der Geest, J. N., Kemner, C., Verbaten, M. N., \& Van Engeland, H. (2002). Gaze behavior of children with pervasive developmental disorder toward human faces: A fixation time study. Journal of Child Psychology and Psychiatry, 43, 669-78.

Yeargin-Allsopp, M., Rice, C., Karapurkar, T., Doernberg, N., Boyle, C., \& Murphy, C. (2003). Prevalence of autism in a US metropolitan area. JAMA, 289, 49-55. 
Fernanda Tebexreni Orsati é doutoranda em Educação Especial pela Syracuse University.

Tatiana Mecca é mestranda do Programa de Pós-graduação em Distúrbios do Desenvolvimento da Universidade Presbiteriana Mackenzie.

José Salomão Schwartzman é Professor Titular do Programa de Pós-graduação em Distúrbios do Desenvolvimento da Universidade Presbiteriana Mackenzie

Elizeu Coutinho de Macedo é Professor do Programa de Pósgraduação em Distúrbios do Desenvolvimento da Universidade Presbiteriana Mackenzie.

Recebido: $16 / 05 / 2008$

$1^{a}$ revisão: 05/02/2009

$2^{a}$ revisão: $28 / 04 / 2009$

$3^{a}$ revisão: $28 / 07 / 2009$

Aceite final: 18/08/2009 